Geometry \& $\mathcal{T}$ opology

Volume 3 (1999) 235-252

Published: 2 September 1999

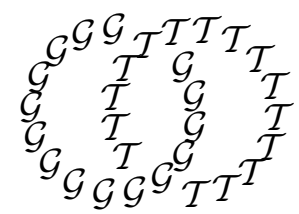

\title{
All two dimensional links are null homotopic
}

\author{
ARthur BARTELS \\ Peter Teichner \\ University of California in San Diego \\ La Jolla, CA, 92093-0112, USA \\ Email: abartels@math.ucsd.edu and teichner@math.ucsd.edu
}

\begin{abstract}
We show that any number of disjointly embedded 2-spheres in 4-space can be pulled apart by a link homotopy, ie, by a motion in which the 2 -spheres stay disjoint but are allowed to self-intersect.
\end{abstract}

AMS Classification numbers Primary: 57Q45

Secondary: 57Q60

Keywords: Link homotopy, Milnor group, concordance

Proposed: Robion Kirby

Seconded: Cameron Gordon, Joan Birman
Received: 21 May 1999

Revised: 27 August 1999

Copyright Geometry and Topology 


\section{Introduction}

In order to separate 3-dimensional linking and knotting phenomena, John Milnor introduced in 1954 the notion of a link homotopy [12]. It is a one-parameter family of maps

$$
S^{1} \amalg \cdots \amalg S^{1} \rightarrow \mathbb{R}^{3}
$$

during which one allows self-intersections but does not allow different components to cross. For example, any knot is link homotopically trivial but the Hopf link is not. Another example of a homotopically essential link in $\mathbb{R}^{3}$ is given by the Borromean rings which are detected by the generalized linking number $\mu(1,2,3)$. More generally, Milnor showed that a link in $\mathbb{R}^{3}$ is homotopically trivial if and only if all its $\mu$-invariants (with non-repeating indices) vanish. Another way to formulate this result is to consider a certain quotient of the fundamental group of the link complement, now known as the Milnor group, which is an invariant of link homotopy (see Section 2). Then a link is homotopically trivial if and only if its Milnor group is isomorphic to the Milnor group $M F_{n}$ of the unlink (with $n$ components). In our paper this particular group $M F_{n}$, the free Milnor group, is used as the key ingredient to prove the following result:

Theorem 1 Every smooth link $L: S^{2} \amalg \cdots \amalg S^{2} \hookrightarrow \mathbb{R}^{4}$ is link homotopic to the unlink.

The beginning of 4-dimensional link homotopy was the paper [6] by Roger Fenn and Dale Rolfsen in 1985 who construct two disjointly immersed 2-spheres in $\mathbb{R}^{4}$ which are not link homotopically trivial. William Massey and Rolfsen had just introduced higher dimensional link homotopy and observed that their generalized linking number for two 2 -spheres in $\mathbb{R}^{4}$ vanishes on embedded links. They ask in [11] whether Theorem 1 is true for two component links, hence this question is sometimes referred to as the Massey-Rolfsen problem. A proof has been attempted several times but as the referee points out, this paper gives the first correct solution, as well as a generalization to arbitrary many components.

In the course of our proof we have to introduce many self-intersections into the components of $L$ but surprisingly we can keep different components disjoint. The argument has two completely independent steps: one is to construct a link concordance to the unlink and the other is to improve the link concordance to a link homotopy.

Both of these steps generalize to links of $n$-spheres in $\mathbb{R}^{n+2}$ for all $n>1$. The first step is Bartels' PhD thesis [1], the second Teichner's habilitation [15]. 
These papers are long and yet unpublished whereas both steps are discussed in full detail in this short note in dimension 4 . We would still like to announce the general result, since it seems to come as a surprise how far the MasseyRolfsen problem can be pushed. For readers interested in high dimensional link homotopy we should mention that the theory was developed by Fenn, Habegger, Hilton, Kaiser, Kirk, Koschorke, Massey, Nezhinsky, Rolfsen and others.

Theorem 2 For $n>1$, every smooth link $L: S^{n} \amalg \cdots \amalg S^{n} \hookrightarrow \mathbb{R}^{n+2}$ is link homotopic to the unlink.

The result for two 2 -spheres in $S^{4}$ is also proven by very different methods in [16]. This paper, which is currently being rewritten, actually gives a complete calculation of the group $L M_{2,2}^{4}$ of link homotopy classes of link maps $S^{2} \amalg S^{2} \rightarrow$ $\mathbb{R}^{4}$. In particular, it implies the two component case of the following conjecture.

Conjecture Theorem 1 still holds if one component of the link $L$ is not embedded (but mapped into $\mathbb{R}^{4}$ disjointly from the other components).

The conjecture is supported by the fact that it holds for one- and two-component links and that all known invariants vanish on links with only one nonembedded component. It is not difficult to construct three disjointly immersed 2 -spheres in $\mathbb{R}^{4}$, one of them embedded, which are not homotopically trivial.

Here is a brief outline of the proof for Theorem 1. The last section (Section 5) contains the proof that "link concordance implies link homotopy" for link maps in dimension 4. The main idea (which works for arbitrary dimensions as long as the codimension is $\geq 2$ ) is to develop a theory of ambient singular handles. This leads to a generalization to immersions of Colin Rourke's ambient handle proof [13] of Hudson's theorem that "concordance implies isotopy" in codimension $\geq 3$. The proof given here simplifies in contrast to higher dimensions out of several reasons:

- there are no triple points,

- the singular handles are given by the well known 4-dimensional Whitney move (and it's reverse),

- there is no need to use ambient handle slides (as in [13]) or ambient Cerf theory (as in [15]) because the relevant product structures are guaranteed by simple arguments involving only 0 -handles.

In the other sections we show that the link $L$ is link concordant to the unlink, ie, that it bounds disjoint immersions of 3 -balls into $D^{5}$. One observes that 
by possibly introducing more singularities (finger moves) into the 3 -balls one may assume that the fundamental group of the complement of these 3 -balls is the free Milnor group $M F_{n}$. Therefore, we first construct a certain 5-manifold with fundamental group $M F_{n}$ which plays the role of the complement of the 3-balls in $D^{5}$ and we then fill it in with standard thickenings of immersed 3balls to get back $D^{5}$. The first step is to ask whether 0 -surgery on $L$ bounds a 5 -manifold over the group $M F_{n}$. The answer to this question is "yes" using the following two known algebraic facts [12], [2] (which will be explained in the next section):

- $M F_{n}$ is a finitely presented nilpotent group.

- The tower of nilpotent quotients of the free group $F_{n}$ is homologically pro-trivial.

By Alexander duality and Stallings' theorem [14], the nilpotent quotients of $\pi_{1}\left(S^{4}-L\right)$ are the same as of $F_{n}$ which will prove the existence of the searched for 5-manifold. The argument finishes with some surgeries which correct the second homology such that the filling actually leads to a 5 -manifold which is recognized to be the 5 -ball by an application of the 6 -dimensional h-cobordism theorem.

Acknowledgments We wish to thank Mike Freedman and Colin Rourke for interesting comments.

The second author is supported by NSF Grant DMS 97-03996.

\section{$2 \quad$ Milnor groups}

We first collect the necessary group theoretic facts: The lower central series of a group $G$ is defined by $G_{1}:=G, G_{k+1}:=\left[G, G_{k}\right]$ for $k \geq 1$.

All nilpotent quotients of a group factor through some $G / G_{k}$, however these are not the only interesting nilpotent quotients. If $G$ is provided with a set of normal generators, $G=\ll x_{1}, \cdots, x_{n} \gg$, then one defines the Milnor group

$$
M G:=G / \ll\left[x_{i}, x_{i}^{y}\right] \gg, \quad i=1, \ldots, n \text { and } y \in G .
$$

These quotients were introduced in Milnor's thesis [12]. The following lemma about Milnor groups uses the fact that we have chosen the elements $x_{i}$ to be normal generators for $G$.

Lemma $1 M G$ has nilpotency class $\leq n$, ie $M G_{n+1}=\{1\}$. 
Proof We will use an induction on the number $n$ of normal generators. If $n=1$ the relations imply that all $x_{i}^{y}$ commute in $M G$. Since by assumption these elements generate $G$ it follows that all commutators vanish in $M G$.

Now assume the statement holds for groups with $n-1$ normal generators and let $G$ be normally generated by $x_{i}, 1 \leq i \leq n$. Define $A_{i} \unlhd M G$ to be the normal closure of the element $x_{i}$. Since all the conjugates of this element commute, $A_{i}$ is abelian. Moreover, the intersection $A$ of all $A_{i}$ lies obviously in the center of $M G$. Now consider a commutator $[x, y]$ with $x \in M G, y \in M G_{n}$. Since all quotients $M G / A_{i}$ are Milnor groups with $n-1$ generators it follows by induction that $y \in A$ and thus $y$ is central ie $[x, y]=1$ in $M G$. This shows that $M G_{n+1}=\{1\}$.

Corollary $2 M G$ is generated by $x_{1}, \ldots, x_{n}$ and is also finitely presented.

Proof The statement for the generators follows from the standard rewriting process in nilpotent groups: If a nilpotent group $N$ is normally generated by $x_{i}$ then it is also generated by these elements. One uses an induction on the nilpotency class of $N$ based on the fact that $x \equiv y \bmod N_{k}$ implies $a^{x} \equiv a^{y}$ $\bmod N_{k+1}$ for all $a, x, y \in N$. Moreover, the fact that $N_{k}$ is generated by $k-$ fold commutators $\left[x_{i_{1}}, \ldots, x_{i_{k}}\right]$ if the $x_{i}$ generate $N$ shows that $N_{k}$ is finitely generated if $N$ is. An induction on the nilpotency class together with the fact that a (central) extension of finitely presented groups is finitely presented implies that a finitely generated nilpotent group is also finitely presented.

We need one more result which is basically due to Bousfield and Kan [2], and also uses some work of Ferry [4] and Cochran [3]. Let $\Omega$ be any generalized homology theory. For a group $G$ we abbreviate the reduced theory by

$$
\widetilde{\Omega}_{k}(G):=\operatorname{Ker}\left(\Omega_{k}(K(G, 1)) \rightarrow \Omega_{k}(*)\right)
$$

where $\pi_{1} K(G, 1)=G$ and $\pi_{i} K(G, 1)=0$ for $i>1$.

Theorem 3 Let $F$ be the free group on $n$ generators. Given $r \in \mathbb{N}$ and $k>1$ such that $\Omega_{k-1}(*)=0$, there exists an integer $d=d(k, r, n)$ such that the map

$$
\widetilde{\Omega}_{k}\left(F / F_{r+d}\right) \longrightarrow \widetilde{\Omega}_{k}\left(F / F_{r}\right)
$$

is trivial.

Proof For $\Omega=$ ordinary homology, the theorem comes from [2]. To get the general statement, one uses an eventual Hurewicz Theorem as in [3]. It is 
therefore necessary to reduce to the case of simply-connected spaces. This can be easily done in our context by picking maps $f_{i}: S^{1} \rightarrow K\left(F / F_{r}, 1\right)$, which represent the generators $x_{i}$ of $F$, and attach 2-cells to get simply-connected complexes $X_{r}$. Then

$$
H_{k}\left(X_{r}\right) \cong H_{k}\left(F / F_{r}\right)
$$

for all $k>1$, so the again by [2] the maps $H_{k}\left(X_{r+d}\right) \rightarrow H_{k}\left(X_{r}\right)$ are eventually zero. By the eventual Hurewicz Theorem for simply-connected spaces it follows that the maps $X_{r+d} \rightarrow X_{r}$ are eventually null homotopic and thus the induced maps $\widetilde{\Omega}_{k}\left(X_{r+d}\right) \rightarrow \widetilde{\Omega}_{k}\left(X_{r}\right)$ are eventually zero. Moreover, there is an exact sequence

$$
\widetilde{\Omega}_{k+1}\left(\bigvee^{n} S^{2}\right) \longrightarrow \widetilde{\Omega}_{k}\left(F / F_{r}\right) \stackrel{i_{*}}{\longrightarrow} \widetilde{\Omega}_{k}\left(X_{r}\right)
$$

By assumption and excision we have

$$
\widetilde{\Omega}_{k+1}\left(\vee^{n} S^{2}\right) \cong \bigoplus^{n} \Omega_{k-1}(*)=0
$$

which proves that $i_{*}$ is a monomorphism. The same exact argument works for $r$ replaced by $r+d$ which implies our claim.

\section{Singular handles}

Let $\Delta_{0}: D^{3} \hookrightarrow D^{5}$ be a standardly embedded slice disk for an unknot $S^{2} \subset S^{4}$. Let $\Delta_{\nu}: D^{3} \leftrightarrow D^{5}$ be obtained from $\Delta_{0}$ by performing $\nu$ finger moves on $\Delta_{0}$ along $\operatorname{arcs} \gamma_{i}$ in the interior of $D^{5}$ connecting pairs of points on $\Delta_{0}$. So $\Delta_{\nu}$ is an immersed slice disk for the unknot, see figure 1. The self intersections of $\Delta_{\nu}$ consist of a disjoint union of $\nu$ circles. Now extend $\Delta_{\nu}$ to a thickening $\widetilde{\Delta_{\nu}}: D^{3} \times D^{2} \rightarrow D^{5}$ and define

$$
\begin{array}{ll}
\Sigma_{\nu} H & :=\widetilde{\Delta_{\nu}}\left(D^{3} \times D^{2}\right) \\
\Sigma_{\nu} F & :=\text { closure of }\left(\partial \Sigma_{\nu} H-S^{4}\right) \\
\Sigma_{\nu}^{+} F & :=\Sigma_{\nu} F \cup_{S^{2} \times S^{1}} D^{3} \times S^{1} \\
\Sigma_{\nu}^{+} H & :=\Sigma_{\nu} H \cup_{S^{2} \times D^{2}} D^{3} \times D^{2} .
\end{array}
$$

Note that $\partial \Sigma_{\nu} H=\Sigma_{\nu} F \cup_{S^{2} \times S^{1}} S^{2} \times D^{2}$ and $\Sigma_{\nu}^{+} F=\partial \Sigma_{\nu}^{+} H . \quad \Sigma_{\nu} H$ can also be constructed (abstractly) as a self-plumbing of $D^{3} \times D^{2}$ along the self intersection circles. Similarly, $\Sigma_{\nu}^{+} H$ is a self-plumbing of $S^{3} \times D^{2}$.

If $P=\Delta_{\nu}(x)$, then $m_{P}:=\widetilde{\Delta_{\nu}}\left(x \times S^{1}\right)$ is called the meridian to $\Delta_{\nu}$ at $P$. Let $P=\Delta_{\nu}(x)=\Delta_{\nu}(y)$ be a self intersection point of $\Delta_{\nu}$. (So at $P$ there are actually two different meridians.) Let $B: \mathbb{R} \times \mathbb{C} \times \mathbb{C} \hookrightarrow D^{5}$ be a parameterization 


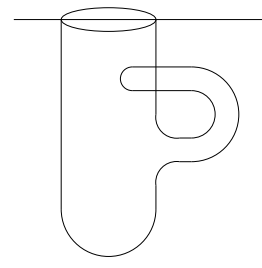

Figure 1: The immersion $\Delta_{1}$

of an open neighborhood of $P$ such that

$$
\begin{array}{llll}
B(0,0,0) & =P & \\
B \cap \Delta_{\nu} & =\mathbb{R} \times \mathbb{C} \times 0 & \cup & \mathbb{R} \times 0 \times \mathbb{C} \\
B \cap \Sigma_{\nu} H & =\mathbb{R} \times \mathbb{C} \times D^{2} & \cup & \mathbb{R} \times D^{2} \times \mathbb{C} .
\end{array}
$$

Then $T_{P}:=B\left(0 \times S^{1} \times S^{1}\right) \subset \Sigma_{\nu} F$ is called the Clifford torus at $P$. Note that $T_{P}$ bounds a solid torus, say $B\left(0 \times S^{1} \times D^{2}\right)$, in $\Sigma_{\nu} H$. Choose an arc in $D^{3}$ connecting $x$ to $y$. The image under $\Delta_{\nu}$ of this arc gives a closed curve in $\Sigma_{\nu} H$ and a choice of an arc connecting this curve to a base point leads to an element in $\pi_{1} \Sigma_{\nu} H$. Applying this procedure to one point of each selfintersection component gives $\nu$ elements $g_{1}, \ldots, g_{\nu} \in \pi_{1} \Sigma_{\nu} H$, and it is not hard to see that $\pi_{1} \Sigma_{\nu} H$ is the free group on $\nu$ generators $g_{i}$. The $g_{i}$ have preimages in $\pi_{1} \Sigma_{\nu} F$ which we will again denote by $g_{i}$. Let $m \in \pi_{1} \Sigma_{\nu} F$ be the element induced by a fixed meridian (to the unknot $S^{2} \subset S^{4}$ say). The existence of the Clifford tori in $\Sigma_{\nu} F$ implies the equations

$$
\left[m, m^{g_{i}}\right]=1 \in \pi_{1} \Sigma_{\nu} F
$$

for $i=1, \ldots, \nu$.

Lemma $3 H_{2} \Sigma_{\nu} F$ maps onto $H_{2} \Sigma_{\nu} H$ and the Clifford tori represent elements in the kernel of this map.

Proof The Mayer-Vietoris sequence

$$
0=H_{3} D^{5} \rightarrow H_{2} \Sigma_{\nu} F \rightarrow H_{2} \Sigma_{\nu} H \oplus H_{2}\left(D^{5}-\Sigma_{\nu} H\right) \rightarrow H_{2} D^{5}=0 .
$$

implies the first statement. The Clifford tori represent trivial elements in $H_{2} \Sigma_{\nu} H$, since they bound solid tori there.

Note that $\pi_{1} \Sigma_{\nu} F \cong \pi_{1} \Sigma_{\nu}^{+} F$ and $\pi_{1} \Sigma_{\nu} H \cong \pi_{1} \Sigma_{\nu}^{+} H$. The meridian is an element of the kernel of $\pi_{1} \Sigma_{\nu}^{+} F \rightarrow \pi_{1} \Sigma_{\nu}^{+} H$. We will now construct a second manifold $W$ bounding $\Sigma_{\nu}^{+} F$ such that $m$ and $g_{1}, \ldots g_{\nu}$ give nontrivial elements in $\pi_{1} W$. 
Let $K_{0}: S^{3} \hookrightarrow S^{5}$ be an unknot. Let $E:=S^{5} \#^{\nu} S^{1} \times S^{4}$ where the connected sum operation is done away from $K_{0}$. So we still have $K_{0}: S^{3} \hookrightarrow E$. Now perform finger moves on $K_{0}$ along $\nu \operatorname{arcs} \beta_{1}, \ldots, \beta_{\nu}$, each following one of the handles just added, to obtain $K_{\nu}: S^{3} \rightarrow E$. Thicken this to $\widetilde{K_{\nu}}: S^{3} \times D^{2} \rightarrow E$. Note that $\widetilde{K_{\nu}}\left(S^{3} \times D^{2}\right)$ is just $\Sigma_{\nu}^{+} H$. Now $W:=E-$ Interior of $\Sigma_{\nu}^{+} H$ is the desired manifold. $W$ is a spin manifold since $E$ is one.

Lemma 4 Let $N \unlhd F\left(m_{0}, b_{1}, \ldots, b_{\nu}\right)$ be normally generated by $\left[m_{0}, m_{0}^{b_{1}}\right], \ldots$, $\left[m_{0}, m_{0}^{b_{\nu}}\right]$. Then there is a homomorphism

$$
\varphi_{0}: \pi_{1} \Sigma_{\nu} F \rightarrow F\left(m_{0}, b_{1}, \ldots, b_{\nu}\right) / N
$$

such that $\varphi(m)=m_{0}$ and $\varphi\left(g_{i}\right)=b_{i}$ for $i=1, \ldots \nu$. Moreover, this map factors through $\pi_{1} \Sigma_{\nu} F \rightarrow \pi_{1} W$ induced from the inclusion $\Sigma_{\nu} F \subset \Sigma_{\nu}^{+} F \hookrightarrow W$.

Proof To simplify notation we will assume $\nu=1$. The general case can be worked out analogously. Let $B: \mathbb{R}^{4} \times(0,4) \hookrightarrow E$ be a parameterization of a neighborhood of $\beta_{1}$ in $E$ such that $B \cap \beta_{1}=0 \times[1,2]$ and $B \cap K_{0}=$ $\left(0 \times \mathbb{R}^{3}\right) \times 1 \cup\left(\mathbb{R}^{3} \times 0\right) \times 2$. We may assume that $B \cap K_{1}=S \cup T$ where

$$
\begin{aligned}
& S=\quad\left(\mathbb{R} \times \mathbb{R}^{2} \times 0\right) \quad \times 2 \\
& T=\quad\left(0 \times \mathbb{R}^{3}-D^{3}\right) \times 1 \\
& \cup\left(0 \times S^{2}\right) \quad \times[1,3] \\
& \cup\left(0 \times D^{3}\right) \times 3 .
\end{aligned}
$$

This can in fact be taken as a definition of a finger move.

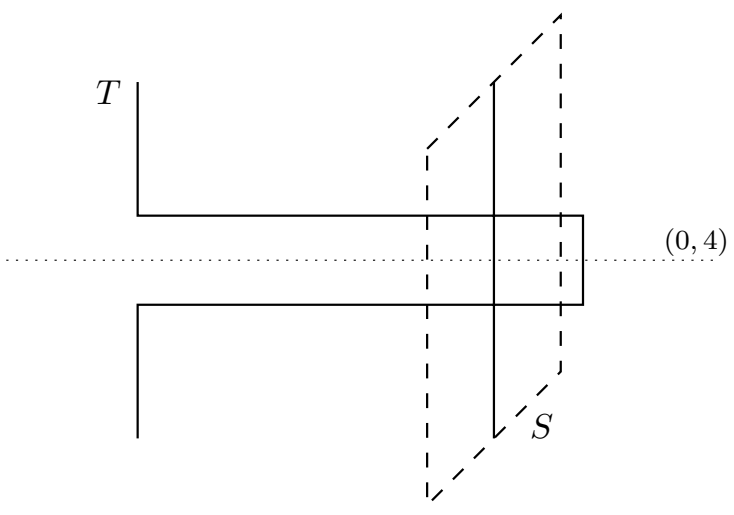

Figure 2: A finger move

Let $\bar{B} \cong D^{5}$ be the compactification of $B$. We have

$$
\left(E-K_{1}\right)=\left(E-\left(K_{1} \cup B\right)\right) \cup_{\partial \bar{B}-K_{1}}\left(\bar{B}-K_{1}\right) .
$$


Note that $\pi_{1}\left(E-\left(K_{0} \cup B\right)\right) \cong \pi_{1}\left(E-K_{0}\right) \cong F\left(m_{0}, b_{1}\right)$, where $m_{0}$ is a meridian to $K_{0}$ and $b_{1}$ is an element corresponding to the handle. $\left(B-K_{1}\right)$ is the complement of two intersecting slice disks $(S$ and $T)$ in the 5 -ball and so by Alexander duality $H_{1}\left(B-K_{1}\right) \cong \mathbb{Z}^{2}$. (Actually $\pi_{1}\left(B-K_{1}\right) \cong \mathbb{Z}^{2}$ since both slice disks are unknotted.) The corresponding map $\pi_{1}\left(B-K_{1}\right) \rightarrow \mathbb{Z}^{2}$ sends meridians $m_{S}$ and $m_{T}$ of $S$ and $T$ to the generators. $\partial \bar{B} \cap(S \cup T)$ is a trivial two component link and thus $\pi_{1}\left(\partial \bar{B}-K_{1}\right) \cong F\left(m_{S}, m_{T}\right)$. We may assume that $\pi_{1}\left(\partial \bar{B}-K_{1}\right) \rightarrow \pi_{1}\left(E-\left(K_{1} \cup B\right)\right)$ sends the meridians to $S$ and $T$ to $m_{0}$ and $m_{0}^{b_{1}}$. Van Kampen's Theorem gives now a map

$$
\pi_{1}\left(E-K_{1}\right) \rightarrow F\left(m_{0}, b_{1}\right) *_{F\left(m_{S}, m_{T}\right)} \mathbb{Z}^{2} \cong F\left(m_{0}, b_{1}\right) /\left[m_{0}, m_{0}^{b_{1}}\right] .
$$

The inclusion $\Sigma_{\nu} F \hookrightarrow\left(E-K_{1}\right)$ sends $m$ to $m_{0}$ and $g_{1}$ to $b_{1}$ (up to some power of $\left.m_{0}\right)$ and so the inclusions $\Sigma_{\nu} F \hookrightarrow W \hookrightarrow\left(E-K_{1}\right)$ induce the desired map.

\section{Singular slice disks for embedded links}

Let $L: S^{2} \amalg \cdots \amalg S^{2} \hookrightarrow S^{4}$ be an embedded link of $n$ components. Let $X^{4}$ be the manifold obtained by surgery on $L$. There is no ambiguity about the framing because $\pi_{2} S O(2)=0$. Note that $X$ bounds $D^{5} \cup_{L \times D^{2}} \amalg^{n} D^{3} \times D^{2}$. The later manifold possesses a unique spin structure and induces therefore one on $X$. Let $\pi$ be the fundamental group of $X$ or equally, of the link complement.

Let $m_{1}, \ldots, m_{n}$ be a choice of meridians to the components of $L$. Let $k \geq n+1$, so by Lemma $1 M F\left(x_{1}, \ldots, x_{n}\right)_{k}=0$. The map $\Phi$ in the following diagram is given by $\Phi\left(x_{i}\right)=m_{i}$. We abbreviate $F:=F\left(x_{1}, \ldots, x_{n}\right)$.

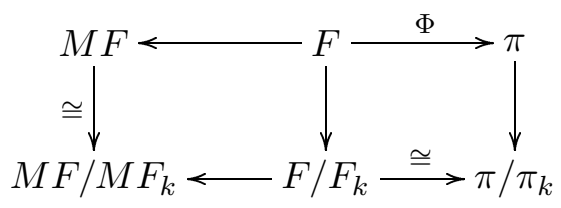

By Alexander Duality $H_{2}\left(S^{4}-L\right)=0$ and hence $H_{2} \pi=0$. Since $H_{1} \pi=$ $H_{1}\left(S^{4}-L\right)$ is freely generated by the meridians, $\Phi$ induces an isomorphism $H_{1} F \cong H_{1} \pi$. So by Stalling's Theorem [14] the isomorphism in the bottom line of the above diagram follows. The diagram gives a map $\Psi: \pi \rightarrow M F$. For any $k \geq n+1$ this map factors as

$$
\pi \rightarrow F / F_{k} \rightarrow F / F_{n+1} \rightarrow M F .
$$


Lemma $5 X$ bounds a spin manifold $A_{0}$ with $\pi_{1} A_{0} \cong M F$ such that $\Psi$ is the induced map on fundamental groups.

Proof Consider $\alpha:=\left[X, \sigma, \Psi_{0}\right] \in \Omega_{4}^{\text {spin }}(M F)$. Here $\sigma$ is the spin structure on $X$ constructed above and $\Psi_{0}: X \rightarrow K(M F, 1)$ is the map that gives $\Psi$ on the fundamental group. Since $[X, \sigma]$ is a spin boundary we have $\alpha \in \widetilde{\Omega}_{4}^{s p i n}(M F)$. The factorization of $\Psi$ implies that $\alpha$ is in the image of the composition

$$
\widetilde{\Omega}_{4}^{\text {spin }}\left(F / F_{k}\right) \rightarrow \widetilde{\Omega}_{4}^{\text {spin }}\left(F / F_{n+1}\right) \rightarrow \widetilde{\Omega}_{4}^{\text {spin }}(M F)
$$

for every $k \geq n+1$. Using $\Omega_{3}^{\text {spin }}(*)=0$, Theorem 3 implies now $\alpha=0$ and hence $X$ bounds a spin manifold $A_{0}$ such that

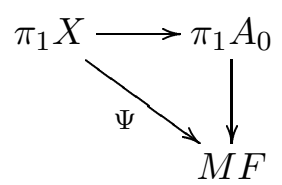

commutes. It is now a standard procedure to do spin structure preserving surgeries on circles in the interior of $A_{0}$ to obtain $\pi_{1} A_{0} \cong M F$.

From Corollary 2 it follows that $M F\left(x_{1}, \ldots, x_{n}\right)$ can be constructed from $F\left(x_{1}, \ldots, x_{n}\right)$ by introducing relations $\left[x_{i}, x_{i}^{h_{i, j}}\right]=1$ for $i=1, \ldots, n$ and $j=1, \ldots, \nu$ and some $h_{i, j} \in F\left(x_{1}, \ldots, x_{n}\right)$. Fix this $\nu$ and the $h_{i, j}$ from now on. Let

$$
Y^{4}=\left(S^{4}-L \times D^{2}\right) \cup_{L \times S^{1}} \amalg^{n} \Sigma_{\nu} F .
$$

So $Y$ is the boundary of $D^{5} \cup_{L \times D^{2}} \amalg^{n} \Sigma_{\nu} H$ (see figure 3). We will enumerate the $n$ copies of $\Sigma_{\nu} F$ in $Y$ as $\Sigma_{\nu} F_{1}, \ldots, \Sigma_{\nu} F_{n}$. We will also write $m, g_{1}, \ldots, g_{n} \in$ $\pi_{1} \Sigma_{\nu} F$ as $m_{i}, g_{i, 1}, \ldots, g_{i, \nu}$ when considered as elements of $\pi_{1} \Sigma_{\nu} F_{i}$. By van Kampen's Theorem we now have

$$
\pi_{1} Y=\pi *_{m_{1}} \pi_{1} \Sigma_{\nu} F_{1} *_{m_{2}} \cdots * m_{n} \pi_{1} \Sigma_{\nu} F_{n} .
$$

Using $\varphi_{0}$ from Lemma 4 we can construct a map $\varphi: \pi_{1} Y \rightarrow M F$ such that $\varphi\left(m_{i}\right)=x_{i}, \varphi\left(g_{i, j}\right)=h_{i, j}$ and $\varphi=\Psi$ on $\pi$.

Lemma $6 Y$ bounds a spin manifold $A$ with $\pi_{1} A \cong M F$ such that $\varphi$ is the induced map on the fundamental group.

Proof Recall that $X=\left(S^{4}-L \times D^{2}\right) \cup_{L \times S^{1}} \amalg^{n} D^{3} \times S^{1}$ is bounded by $A_{0}$ by Lemma 5 . Let $W_{1}, \ldots, W_{n}$ be $n$ copies of $W$, the manifold from Lemma 4 . 

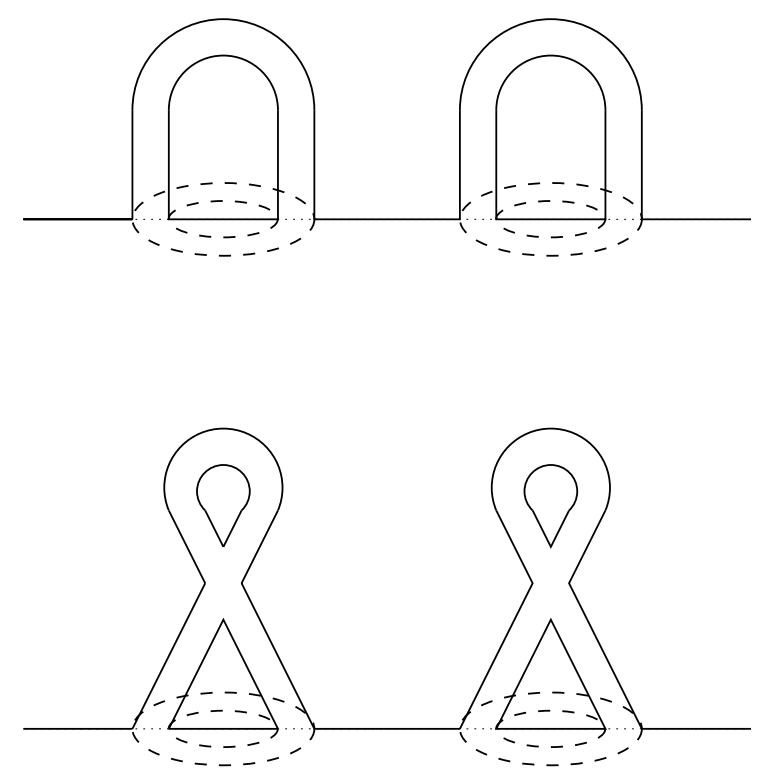

Figure 3: Attaching handles to obtain $X$ and $Y$

We may write $\partial W_{i}=\Sigma_{\nu}^{+} F_{i}=\Sigma_{\nu} F_{i} \cup_{S^{2} \times S^{1}} D^{3} \times S^{1}$. Now we glue $A_{0}$ to $\amalg_{i} W_{i}$ along parts of their boundary to obtain a manifold

$$
A:=A_{0} \cup_{\amalg^{n} D^{3} \times S^{1}} \amalg_{i} W_{i}
$$

with boundary $Y$.

$$
\pi_{1} A=\pi_{1} A_{0} *_{m_{1}} \pi_{1} W_{1} *_{m_{2}} \cdots *_{m_{n}} \pi_{1} W_{n} .
$$

Using Lemma 4 and $\pi_{1} A_{0} \cong M F$ we can find a map $\pi_{1} A \rightarrow M F$ making the triangle

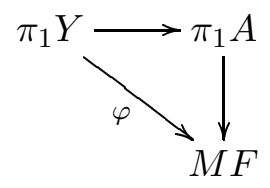

commute. By the Mayer-Vietoris sequence

$$
H^{2}\left(A ; \mathbb{Z}_{2}\right) \cong H^{2}\left(A_{0} ; \mathbb{Z}_{2}\right) \oplus H^{2}\left(W_{1} ; \mathbb{Z}_{2}\right) \oplus \cdots \oplus H^{2}\left(W_{n} ; \mathbb{Z}_{2}\right)
$$

All manifolds on the right hand side are spin and have therefore vanishing second Stiefel-Whitney class. Hence $A$ is also spin. Again we can do spin structure preserving surgeries in the interior of $A$ to obtain $\pi_{1} A \cong M F$.

Theorem $4 \quad L$ bounds disjointly immersed slice disks in $D^{5}$. 
Proof Let $A$ be the spin manifold obtained in Lemma 6. Recall that

$$
\partial A=Y=\left(S^{4}-L \times D^{2}\right) \cup_{L \times S^{1}} \amalg_{i} \Sigma_{\nu} F_{i}
$$

Now let $\Sigma H:=\Sigma_{\nu} H_{1} \amalg \cdots \amalg \Sigma_{\nu} H_{n}$ be the disjoint union of $n$ copies of $\Sigma_{\nu} H$. Then $\partial \Sigma H$ and $\partial A$ both contain the disjoint union of $n$ copies of $\Sigma_{\nu} F$ which we will denote by $\Sigma F$. Now glue $\Sigma H$ to $A$ along $\Sigma F$ to obtain

$$
D:=A \cup_{\Sigma F} \Sigma H \text {. }
$$

Then $\partial D=\left(S^{4}-L \times D^{2}\right) \cup_{L \times S^{1}}\left(L \times D^{2}\right)=S^{4}$ and $L$ bounds disjointly immersed slice disks in $\Sigma H \subset D$. We will modify $D$ to the 5 -ball without destroying the immersed slice disks. In order to do so it is enough to make $D$ 2-connected (using Poincaré duality and the $\mathrm{h}$-cobordism Theorem). Again by van Kampen's Theorem

$$
\pi_{1} D=\pi_{1} A *_{\pi_{1} \Sigma_{\nu} F_{1}} \pi_{1} \Sigma_{\nu} H_{1} *_{\pi_{1} \Sigma_{\nu} F_{2}} \cdots *_{\pi_{1} \Sigma_{\nu} F_{n}} \pi_{1} \Sigma_{\nu} H_{n}
$$

and this group is normally generated by the meridians $m_{i}$ (the $g_{i, j}$ are identified with products of meridians). But the meridians bound 2-disks in $\Sigma H$ and so $\pi_{1} D=1$. To kill $\pi_{2} D$ we will do surgery on 2 -spheres in $A$ (and hence away from the immersed slice disks).

The horizontal line of the diagram below is part of a Mayer-Vietoris sequence. By Lemma $4 H_{1} \Sigma F \cong H_{1} A \oplus H_{1} \Sigma H$, and this explains that $H_{2} D \rightarrow H_{1} \Sigma F$ below is trivial.

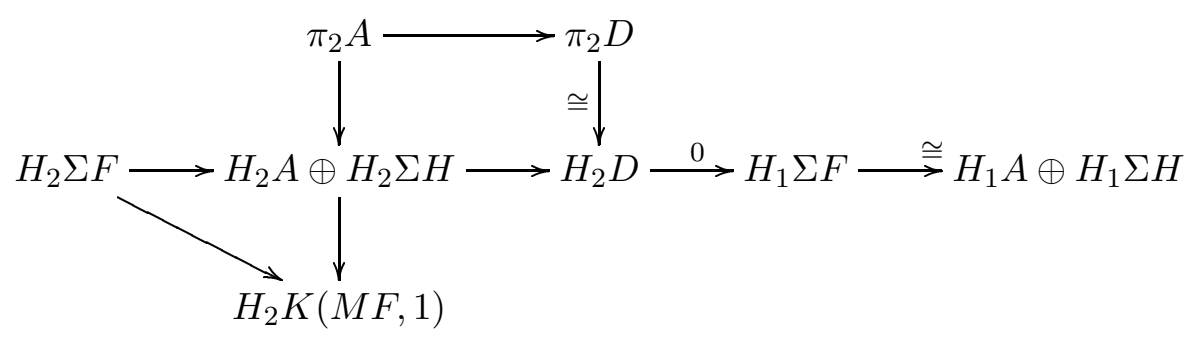

Now according to Lemma $3 \mathrm{H}_{2} \Sigma F$ maps onto $\mathrm{H}_{2} \Sigma H$ and the Clifford tori represent elements in the kernel of this map. But the Clifford tori also generate $H_{2} K(M F, 1)$ [7], and so $H_{2} \Sigma F$ maps also onto $H_{2} K(M F, 1)$. The exact sequence

$$
\pi_{2} A \rightarrow H_{2} A \rightarrow H_{2} K(M F, 1) \rightarrow 0
$$

proves now that $\pi_{2} A$ maps onto $\pi_{2} D \cong H_{2} D$.

It is a classical result of Milnor and Kervaire in [9] that $D$ can be changed to the 5 -ball by a sequence of surgeries on classes in $\pi_{2} D$. (Their original 
formulation considers framed manifolds, but here a spin structure is enough to guarantee trivial normal bundle on 2 -spheres.) We just saw that $\pi_{2} A$ maps onto $\pi_{2} D$, so we can represent these classes by 2 -spheres in $A$ (and by general position embedding these spheres comes for free). So we can do surgery to $A$ and change $D$ to the 5 -ball.

\section{Link concordance implies link homotopy}

Let $M^{3}$ be a 3 -manifold with boundary $\partial M=\partial_{0} M \amalg \partial_{1} M$. Consider a generic immersion $f: M \leftrightarrow N^{4} \times I$, where $N$ is a closed 4 -manifold and $f^{-1}\left(N^{4} \times\{i\}\right)=\partial_{i} M$ for $i=0,1$. The genericity of $f$ implies that the double point set $S(f)$ is a 1-dimensional manifold with boundary in $\partial M$. We say that $f$ is a Morse immersion if $p_{2} \circ f$ is a generic Morse function on $M$ and so is the restriction to $S(f)$. Recall that Morse functions are generic iff all critical values are distinct. For a Morse immersion we also assume that the critical values of $p_{2} \circ f$ and $\left.p_{2} \circ f\right|_{S(f)}$ are distinct. Regular homotopies of a closed surface $F^{2}$ give examples of Morse immersions $F^{2} \times I \rightarrow N^{4} \times I$ without critical points for $p_{2} \circ f$.

Let $g: F^{2} \rightarrow N^{4}$ be a generic immersion. We describe six ways to construct a Morse immersion $f: M \leftrightarrow N^{4} \times I$ with $\partial_{0} M=F$ and $f_{0}=g$ using a guiding map $\alpha$. To simplify the discussion we will ignore framing data on $\alpha$ in these examples. They turn out to be irrelevant for our discussion.

(h1) Let $\alpha: I \hookrightarrow N^{4}$ be guiding arc for a fingermove on $g$. The fingermove along $\alpha$ gives a Morse immersion with $M=F \times I$ and $f_{1}=$ result of a fingermove on $g$.

( $h 2$ ) Let $\alpha: D^{2} \hookrightarrow N^{4}$ be a Whitney disk for $g$. The Whitney move along $\alpha$ gives a Morse immersion with $M=F \times I$ and $f_{1}=$ result of a Whitney move on $g$.

(b0) Let $\alpha: D^{0} \hookrightarrow N^{4}$ be a point in the complement of $g$. Let $h: D^{3} \hookrightarrow N^{4}$ be an embedding into a regular neighborhood of $\alpha$. This data gives rise to Morse immersion with $M=(F \times I) \amalg D^{3}, \partial_{1} M=F \amalg S^{2}$ and $f_{1}=\left.g \amalg h\right|_{S^{2}}$.

(b1) Let $\alpha: D^{1} \hookrightarrow N^{4}$ be an embedded arc such that $\alpha\left(D^{1}\right) \cap g(F)=\alpha\left(\partial D^{1}\right)$ and that $\alpha$ misses all double points of $g$. We can use $\alpha$ to do ambient surgery on $g$ to obtain a Morse immersion with $M=F \times I \cup 1$-handle and $\partial_{1} M=$ result of a surgery on an $S^{0} \subset F$. 
(b2) Let $\alpha: D^{2} \hookrightarrow N^{4}$ be an embedding such that $\alpha\left(D^{2}\right) \cap g(F)=\alpha\left(\partial D^{2}\right)$ and that $\alpha$ misses all double points of $g$. We can use $\alpha$ to do ambient surgery on $g$ to obtain a Morse immersion with $M=F \times I \cup 2$-handle and $\partial_{1} M=$ result of surgery on an $S^{1} \subset F$.

(b3) Let $\alpha: D^{3} \hookrightarrow N^{4}$ be an embedding such that $\left.\alpha\right|_{S^{2}}$ is the restriction of $g$ to one component of $F$ and the interior of $\alpha\left(D^{3}\right)$ misses $g$. This gives a Morse immersion with $M=F \times I \amalg D^{3}, \partial_{1} M \amalg S^{2}=F$ and $f_{1}=\left.g\right|_{F-S^{2}}$.

We will call such Morse immersions elementary and will refer to $h 1, \ldots, b 3$ as the type of an elementary Morse immersion. For type $h 1$ and $h 2$ the Morse function $p_{2} \circ f$ has no critical points whereas the restriction to $S(f)$ has a minimum for $h 1$ and a maximum for $h 2$. The corresponding regular homotopies are the finger move (for $h 1$ ) and the Whitney move (for $h 2$ ).

In the other cases the restriction to $S(f)$ has no critical points but $p_{2} \circ f$ has exactly one critical point of index $i$ for $b i$. These describe the ambient handle decomposition of the 3-manifold $M$.

By turning the interval $I$ upside down, every elementary Morse immersion $f: M \leftrightarrow N^{4} \times I$ can also be reconstructed from $f_{1}: \partial_{1} M \rightarrow N^{4} \times\{1\}$ and an attaching map $\beta$ into $N^{4} \times\{1\}$. This changes the types as follows : $h 1 \leftrightarrow$ $h 2, b 0 \leftrightarrow b 3, b 1 \leftrightarrow b 2$.

Note that in each elementary Morse immersion everything happens in a regular neighborhood of $\alpha \times I \subset N^{4} \times I$. In particular, the complement of this neighborhood is a product (with respect to the product structure on $N^{4} \times I$ ).

The next lemma states that Morse immersions are generic and that every Morse immersion can be constructed from elementary ones. We believe this general position result is well known, details can be found in [15] (for all dimensions). In the following, an isotopy of immersions is the conjugation of the map by diffeotopies of range and domain, just as in the stability condition for smooth mappings [8, Section III].

Lemma 7 (General Position) Let $f: M^{3} \rightarrow N^{4} \times I$ be a generic immersion. It is isotopic (rel $\partial$ ) to a Morse immersion $g: M \hookrightarrow N^{4} \times I$. After a further isotopy, we may assume that there are values $0=a_{0}<a_{1}<\cdots<a_{r}=1$ such that the restrictions $g^{i}$ of $g$ to $\left(p_{2} \circ g\right)^{-1}\left[a_{i}, a_{i+1}\right]$ are elementary Morse immersions (after rescaling $\left[a_{i}, a_{i+1}\right]$ to $[0,1]$ ).

Note that the index $i$ of the elementary Morse immersion $b i$ is the dimension of the guiding map (or descending manifold) $\alpha$ and thus it is consistent to give $h i$ index $i$ as well. This orders all the critical points of a Morse immersion. 
Lemma 8 (Reordering) In the notation of Lemma 7, if index $g^{i+1} \leq$ index $g^{i}$, then $g$ is isotopic to a Morse immersion $h: M^{3} \rightarrow N^{4} \times I$ such that each $h^{i}$ is elementary and

(i) $g^{j}=h^{j}$ for $j \neq i, i+1$;

(ii) type $h^{i}=$ type $g^{i+1}$ and type $h^{i+1}=$ type $g^{i}$.

Proof Let $\alpha_{i+1}$ (resp. $\beta_{i}$ ) be the attaching maps into $N^{4} \times\left\{a_{i+1}\right\}$ that guide the construction of $g^{i+1}$ (resp. $g^{i}$ ) upwards (resp. downwards) from $g_{0}^{i+1}=g_{1}^{i}$. Now index $g^{i+1} \leq$ index $g^{i}$ implies

$$
\operatorname{dim} \alpha_{i+1}+\operatorname{dim} \beta_{i}=\operatorname{index} g^{i+1}+\left(3-\operatorname{index} g^{i}\right) \leq 3 .
$$

By general position we may assume that $\alpha_{i+1}$ and $\beta_{i}$ are disjoint in the level $N^{4} \times\left\{a_{i+1}\right\}$. But this means that $g^{i}$ and $g^{i+1}$ are independent in the following sense: $\alpha_{i+1}$ can be pushed down to an embedding $\alpha_{i}^{\prime}$ into $N^{4} \times\left\{a_{i}\right\}$ that does not intersect the attaching map $\alpha_{i}$ for $f^{i}$ into $N^{4} \times\left\{a_{i}\right\}$. Now first $h^{i}$ is constructed from $\alpha_{i}^{\prime}$ and then $h^{i+1}$ from $\alpha_{i}$.

Recall that a link map is a continuous map which keeps distinct components disjoint. A link concordance is a link map $f: F \times I \rightarrow N \times I$ such that $f^{-1}(N \times\{i\})=F \times\{i\}$ for $i=0,1$. Finally, a link homotopy is a homotopy through link maps. We make a very simple, but useful observation: If a link concordance $f$ is a product on all but one component, then $p_{1} \circ f$ is a link homotopy. By applying the above general position and reordering lemmas, we will repeatedly be able to apply this observation to prove the main result of this section:

Theorem 5 (Link concordance implies link homotopy) If $f: F^{2} \times I \rightarrow N^{4} \times$ $I$ is a link concordance then there is a link homotopy $h: F^{2} \times I \rightarrow N^{4}$ such that $f_{i}=h_{i}$ for $i=0,1$.

Proof Using Thom's jet transversality theorem [8, Sections II.4-5] we can assume that $f$ is a generic immersion except for a finite number of cross caps [8, page 179]. As in Whitney's original immersion argument, these cross caps can be pushed off the, say, lower boundary $N \times\{0\}$. This changes the lower boundary $f_{0}$ by cusp homotopies which we may assume are small enough such that they don't change the link homotopy class of $f_{0}$.

Let $g: F^{2} \times I \leftrightarrow N^{4} \times I$ be a Morse immersion satisfying the conclusion from Lemma 7. If all the $g^{i}$ are of types $h 1$ and $h 2$ then $p_{1} \circ g$ is in fact a link 
homotopy and we are done. Let $C_{1}, \ldots, C_{n}$ be the components of $F^{2} \times I$. We know that $C_{1} \cong M^{2} \times I$. Consequently, all critical points of index 0 of $p_{2} \circ g$ on $C_{1}$ can be canceled (by Morse cancellation) by critical points of index 1 . By applying Lemma 8 several times to move other critical points up respectively down, we may assume that the corresponding elementary Morse immersions for the canceling 0 - and 1-handles of $C_{1}$ are consecutive. Let's say they are $g^{k}, g^{k+1}, \ldots, g^{l}$ and let $W:=g^{-1}\left(\left[a_{k}, a_{l+1}\right]\right)$. Then $W \cap C_{1} \cong M^{2} \times I$ and $\left.g\right|_{W}: W \leftrightarrow N^{4} \times\left[a_{k}, a_{l+1}\right]$ is a product on $W-C_{1}$. Hence the observation from above applies and we can replace $g^{k}, g^{k+1}, \ldots, g^{l}$ by elementary Morse immersions of type $h 1$ and $h 2$. More precisely, we replace

$$
g(m, t)=\left(g_{1}(m, t), g_{2}(m, t)\right), \quad t \in\left[a_{k}, a_{l+1}\right]
$$

by $\left(g_{1}(m, t), t\right)$ which doesn't change anything away from $W$ and removes the critical points on $W$. Finally, we can make this map generic, producing a Morse immersion with singularities of types $h 1$ and $h 2$ only.

We can apply the same procedure to all components and all $g^{i}$ of type $b 0$ and (by symmetry) b3. Hence we may now assume that all the $g^{i}$ are of type $h 1, h 2, b 1$ and $b 2$. Again by Lemma 8 we can order the types of the $g^{i}$, such that $g^{k}, g^{k+1}, \ldots, g^{l}$ are consecutive elementary Morse immersions that induce critical points on $C_{1}$. We still know $C_{1} \cong M^{2} \times I$ and the same argument as before allows us to replace $g^{k}, g^{k+1}, \ldots g^{l}$ by elementary Morse immersions of type $h 1$ and $h 2$. Repeating this procedure on $C_{2}, \ldots, C_{n}$ finishes the proof.

Remark 1 The first part of the argument which makes $f$ a generic immersion is not really necessary to prove our Theorem 1 because the output of the previous sections is already a generic immersion by construction. We just included this step for completeness.

Remark 2 The above argument does not explicitly mention handle cancellation except in the elementary case involving 0 -handles, ie, critical points of type $b 0$ (and $b 3$ ). This is done on purpose because only in this simplest case can one avoid to use ambient handle slides in order to obtain canceling pairs of handles. However, if one is willing to introduce a gradient-like vector field more explicitly into the discussion, then handle slides are well defined and can be in fact done ambiently. This is used by Rourke in [13] to do all the steps of the proof of the $\mathrm{h}$-cobordism theorem ambiently, obtaining a proof of Hudson's "concordance implies isotopy" in codimension $\geq 3$ (this restriction is explained in the next remark). Rourke's argument works for embeddings and is generalized to generic immersions in [15], where the multiple point stratification on 
the range of a generic immersion is considered and stratified versions of Morse functions, gradient-like vector fields and handles are introduced.

Remark 3 The reason why Hudson's theorem does not work in codimension 2 can already be seen for knotted arcs in $D^{2} \times I$. More precisely, if one tries to ambiently cancel two handles which do cancel abstractly, one has to push the core $C$ of the higher handle together with the cocore $Q$ of the lower handle into the middle level (this uses the gradient-like vector field). A dimension count shows that they may be assumed disjoint in codimension $\geq 3$ and hence can be canceled ambiently. However, in codimension 2 one can only assume that $C$ and $Q$ intersect transversaly in a finite number of points. This produces basically all possible knotting phenomena. But since $C$ and $Q$ are part of the same component, it is not a problem in the link homotopy world: One just maps the abstract cancellation forward into a neighborhood of $C \cup Q$, producing a link homotopy (rel. boundary) to the situation where $C$ and $Q$ have canceled. By thickening into the dual dimensions of $C$ and $Q$ one sees that each intersection point in $C \cap Q$ contributes to a small sphere of self-intersections on the relevant component. In the dimension range of Theorem $5 C$ and $Q$ are 2-disks and the new self-intersections are circles of double points. Hence in addition to removing a single pair of critical points of types $b 1$ and $b 2$ the procedure introduces one local pair of critical points of type $h 1$ and $h 2$ for each point in $C \cap Q$. This together with Remark 1 explains how the proof of Theorem 1 could be given completely in the category of generic immersions.

Remark 4 In the argument of [13] explained above one has to assume that the dimension range is such that the Whitney trick can be applied. Since this excludes some very interesting low dimensions, the point of view in [15] is different. There ambient Cerf theory is used to give a proof of Hudson's theorem in all dimensions. This method also avoids the explicit mentioning of handle slides but nevertheless the stratified versions of gradient like vector fields are essential.

Remark 5 In [15] it is proven that a link concordance $M^{m} \times I \leftrightarrow N^{n} \times I$, which is an immersion, is homotopic (rel. boundary) to a link homotopy if $m \leq n-2$. The higher dimensional analogues of cross caps, ie, points where the map is not an immersion, are not discussed. However, by Hirsch-Smale immersion theory, one still obtains that link concordance implies link homotopy for maps $S^{m_{1}} \amalg \cdots \amalg S^{m_{r}} \rightarrow S^{n}$ if $m_{i} \leq n-2$. 


\section{References}

[1] A Bartels, Link homotopy in codimension two, PhD thesis, UC San Diego (1999)

[2] A Bousfield, D Kan, Homotopy limits, completions and localizations, Springer Lecture Note 304 (1972)

[3] T Cochran Link Morse immersion invariants and homotopy theory, Invent. Math. 90, (1987) 635-645

[4] S Ferry, Homotoping $\epsilon$-maps to homeomorphisms, Am. J. Math. 101 (1979) 567-582

[5] M H Freedman, F Quinn, The topology of 4-manifolds, Princeton Math. Series 39, Princeton, NJ (1990)

[6] R Fenn, D Rolfsen, Spheres may link in 4-space, J. London Math. Soc. 34 (1986) 177-184

[7] M Freedman, P Teichner, 4-Manifold Topology I: Subexponential Groups, Invent. Math. 122 (1995) 509-529,

[8] M Golubitsky, V Guillemin, Stable mappings and their singularities, Graduate Texts in Mathematics 14, Springer-Verlag (1973)

[9] M Kervaire, J Milnor, Groups of homotopy spheres I, Annals of Math. 77 (1963) 504-537

[10] P Kirk, Link maps in the 4-sphere, Proc. Siegen Topology Symposium, Springer Lecture Note 1350 (1988) 31-43

[11] W Massey, D Rolfsen, Problem Section, from: "Low-dimensional topology", Papers from the third topology seminar held at the University of Sussex, Chelwood Gate, (Roger Fenn, editor) London Math. Soc. Lecture Note Series, 95, Cambridge University Press, Cambridge-New York (1985)

[12] J Milnor, Link groups, Annals of Math. 59 (1954) 177-195

[13] C P Rourke, Embedded handle theory, concordance and isotopy, from: "Topology of manifolds", Proc. of the Georgia Topology Conference 1969, (Cantrell and Edwards, editors) Markham, Chicago (1970) 431-438

[14] J Stallings, Homology and central series of groups, J. Algebra, 2 (1965) 170181

[15] P Teichner, Stratified Morse theory and link homotopy, in preparation

[16] P Teichner, Pulling apart 2-spheres in 4-space, preprint (1997), corrected version to appear 